\title{
Erratum to: Comparison of cisplatin and 5-fluorouracil chemotherapy protocols combined with concurrent radiotherapy for esophageal cancer
}

\author{
Toru Sakayauchi $\cdot$ Kenji Nemoto $\cdot$ Chikashi Ishioka \\ Hiroshi Onishi $\cdot$ Michinori Yamamoto \\ Tomoko Kazumoto $\cdot$ Masaoki Makino $\cdot$ Ryuji Yonekura \\ Jun Itami $\cdot$ Shigeru Sasaki $\cdot$ Gen Suzuki \\ Naofumi Hayabuchi · Hiroyasu Tamamura \\ Rikiya Onimaru $\cdot$ Tetsuro Tamamoto $\cdot$ Shogo Yamada
}

(C) Japan Radiological Society 2009

\section{Erratum to: Jpn J Radiol (2009) 27:131-137 \\ DOI 10.1007/s11604-008-0309-9}

The name and affiliation of an author of the article cited on p. 131 was inadvertently omitted. The corrected list of authors and their affiliations is in the footnote below.

Additionally there is an error on p. 133, on lines 3-2 from the bottom of the left column, "from the study before treatment began" should be "from the study before or during treatment".

The online version of the original article can be found under doi 10.1007/s11604-008-0309-9

T. Sakayauchi $(\bowtie) \cdot$ S. Yamada

Department of Radiation Oncology, Tohoku University School of Medicine, 1-1 Seiryomachi, Aoba-ku, Sendai 980-8574, Japan Tel. +81-22-717-7312; Fax +81-22-717-7316

e-mail: sakayauchi-rad@umin.ac.jp

K. Nemoto

Department of Radiation Oncology, Yamagata University

Faculty of Medicine, Yamagata, Japan

C. Ishioka

Department of Clinical Oncology, Tohoku University School of

Medicine, Sendai, Japan

H. Onishi

Department of Radiology, Faculty of Medicine, University of Yamanashi, Yamanashi, Japan

M. Yamamoto

Department of Radiation Oncology, Kure Medical Center and

Chugoku Cancer Center, Hiroshima, Japan

T. Kazumoto

Department of Radiology, Saitama Cancer Center, Saitama, Japan
M. Makino $\cdot$ R. Yonekura

Department of Radiology, Kagoshima Medical Center, Kagoshima, Japan

J. Itami

Department of Radiation Therapy and Oncology, International Medical Center of Japan, Tokyo, Japan

S. Sasaki

Department of Radiology, Shinshu University School of Medicine, Matsumoto, Japan

G. Suzuki $\cdot$ N. Hayabuchi

Department of Radiology, Kurume University School of Medicine, Kurume, Japan

H. Tamamura

Department of Nuclear Medicine, Fukui Prefectural Hospital, Fukui, Japan

R. Onimaru

Department of Radiology, Hokkaido University Graduate

School of Medicine, Sapporo, Japan

T. Tamamoto

Department of Radiation Oncology, Nara Medical University School of Medicine, Kashihara, Japan 